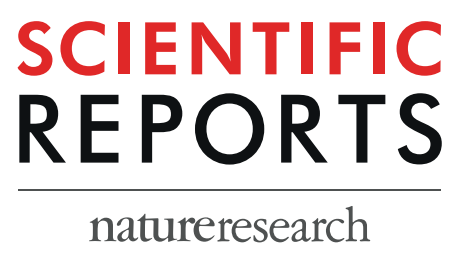

\title{
OPEN Podocyte hypertrophic stress and detachment precedes hyperglycemia or albuminuria in a rat model of obesity and type 2 diabetes-associated nephropathy
}

\author{
Akihiro Minakawa $a^{1,3,6}$, Akihiro Fukuda ${ }^{1,2,6 *}$, Yuji Sato $^{1}$, Masao Kikuchi ${ }^{1,3}$, Kazuo Kitamura ${ }^{3}$, \\ Roger C. Wiggins ${ }^{4,7}$ \& Shouichi Fujimoto ${ }^{1,5,7}$
}

Type2 diabetes-associated nephropathy is the commonest cause of renal failure. Mechanisms responsible are controversial. Leptin-deficient hyperphagic Zucker (fa/fa) rats were modeled to test the hypothesis that glomerular enlargement drives podocyte hypertrophic stress leading to accelerated podocyte detachment, podocyte depletion, albuminuria and progression. By 6 weeks, prior to development of either hyperglycemia or albuminuria, fa/fa rats were hyperinsulinemic with high urinary IGF1/2 excretion, gaining weight rapidly, and had 1.6-fold greater glomerular volume than controls $(P<0.01)$. At this time the podocyte number per glomerulus was not yet reduced although podocytes were already hypertrophically stressed as shown by high podocyte phosphor-ribosomal $\mathrm{S6}$ (a marker of $m$ TORC1 activation), high urinary pellet podocin:nephrin mRNA ratio and accelerated podocyte detachment (high urinary pellet podocin:aquaporin $2 \mathrm{mRNA}$ ratio). Subsequently, fa/fa rats became both hyperglycemic and albuminuric. $24 \mathrm{hr}$ urine albumin excretion correlated highly with decreasing podocyte density $\left(R^{2}=0.86\right)$, as a consequence of both increasing glomerular volume $\left(R^{2}=0.70\right)$ and decreasing podocyte number $\left(R^{2}=0.63\right)$. Glomerular podocyte loss rate was quantitatively related to podocyte detachment rate measured by urine pellet mRNAs. Glomerulosclerosis occurred when podocyte density reached $<50 / 10^{6} \mathrm{um}^{3}$. Reducing food intake by $40 \%$ to slow growth reduced podocyte hypertrophic stress and "froze" all elements of the progression process in place, but had small effect on hyperglycemia. Glomerular enlargement caused by high growth factor milieu starting in pre-diabetic kidneys appears to be a primary driver of albuminuria in fa/fa rats and thereby an under-recognized target for progression prevention. Progression risk could be identified prior to onset of hyperglycemia or albuminuria, and monitored non-invasively by urinary pellet podocyte mRNA markers.

Obesity and type 2 diabetes mellitus prevalence continues to increase worldwide reflecting population access to high energy food and reduced calorie expenditure necessary to sustain life $\mathrm{f}^{1-3}$. Diabetes-associated end stage kidney disease (ESKD) is the commonest cause of renal mortality with attendant human and monetary costs ${ }^{1-4}$.

Micro-albuminuria is recognized as an early marker for both obesity and diabetes-associated nephropathy (DAN), with progressive disease being associated with increasing albuminuria, progressive glomerulosclerosis, and accumulating nephron loss eventually culminating in $\mathrm{ESKD}^{5}$. Diverse mechanisms contribute to nephron loss including injuries to glomerular, tubule-interstitial and vascular compartments ${ }^{6,7}$.

\footnotetext{
${ }^{1}$ Division of Nephrology, Department of Internal Medicine, Faculty of Medicine, University of Miyazaki, Miyazaki, Japan. ${ }^{2}$ Department of Endocrinology, Metabolism, Rheumatology and Nephrology, Faculty of Medicine, Oita University, Yufu, Japan. ${ }^{3}$ First Department of Internal Medicine, University of Miyazaki, Miyazaki, Japan. ${ }^{4}$ Division of Nephrology, Department of Internal Medicine, University of Michigan, Ann Arbor, Michigan, USA. ${ }^{5}$ Department of Hemovascular Medicine and Artificial Organs, University of Miyazaki, Miyazaki, Japan. ${ }^{6}$ These authors contributed equally: Akihiro Minakawa and Akihiro Fukuda. ${ }^{7}$ These authors jointly supervised this work: Roger C. Wiggins and Shouichi Fujimoto. *email: akifukuda@oita-u.ac.jp
} 
Normal podocyte density (number per glomerular tuft volume) is necessary to maintain high resolution glomerular filtration through complete coverage of the filtration surface area by foot processes ${ }^{8}$. At the same time podocytes have limited capacity for replacement such that critical reduction of podocyte density (caused by increased glomerular volume and/or reduced podocyte number) is associated with proteinuria, glomerulosclerosis and ESKD in all progressive glomerular diseases so far examined ${ }^{8-16}$.

In type 1 and type 2 diabetes in man reduced podocyte density is associated with both glomerular enlargement and reduced podocyte number ${ }^{17-19}$. Podocyte injury is present at an early stage and podocyte depletion occurs with advanced DAN ${ }^{17-19}$. Animal model studies implicate a role for mTORC1, the major integrator of growth signaling, in driving podocyte and glomerular injury that reproduces many features associated with progressive DAN in $\operatorname{man}^{20,21}$.

We recently reported that increased glomerular volume and impaired podocyte capacity to adapt to hypertrophic stress are both necessary to cause proteinuria and progressive glomerulosclerosis in a non-diabetic rat model $^{12,15}$. Furthermore, reducing the rate of glomerular growth by calorie intake reduction, even when started late and by modest amounts, prevented downstream consequences manifest by podocyte depletion, proteinuria and glomerulosclerosis. Since glomerular enlargement is well-documented in diabetes, and reducing food intake is a therapy available worldwide, we tested the hypothesis that glomerular enlargement could also drive albuminuria and progression in the Zucker rat model of type 2 diabetes. In this model homozygous (fa/fa) leptin deficiency causes loss of inhibitory feedback for satiety thereby resulting in primary hyperphagia that drives the cardinal features of type 2 diabetes including body weight gain, hyperinsulinemia, peripheral insulin resistance, hyperglycemia and glycosuria. The model also develops progressive albuminuria and glomerulosclerosis, and thereby parallels many aspects of both obesity and type2 DAN in man ${ }^{22-24}$.

\section{Results}

Time-course. Homozygous fa/fa rats were already significantly heavier than $\mathrm{Fa} / \mathrm{fa}$ controls by 6 weeks of age and continued to gain weight faster than control rats prior to onset of overt hyperglycemia (Fig. 1A). In association with persistent high level glycosuria and by 18 weeks fa/fa rat weight had plateaued and by 30 weeks body weight had decreased to below that of $\mathrm{Fa} / \mathrm{fa}$ control which continued weight gain through 46weeks of observation. By 10weeks fa/fa rats had developed overt hyperglycemia (Fig. 1B) associated with glycosuria (Fig. 1C), and increased urine volume due to glycosuria-associated osmotic diuresis (Fig. 1D). $24 \mathrm{hr}$ urine albumin excretion in $\mathrm{fa} / \mathrm{fa}$ rats became significantly increased by 10 weeks, continued to increase to high levels by 38 weeks and plateaued thereafter (Fig. 1E,F). Significant albuminuria did not develop in Fa/fa control rats. Blood pressure measured by tail cuff remained in the normal range (systolic $110-130 \mathrm{mmHg}$ ) with no significant difference between $\mathrm{fa} / \mathrm{fa}$ and $\mathrm{Fa} / \mathrm{fa}$ controls at any time point (data not shown).

Histologic changes and morphometry. Fig. 1G,H, Supplemental Fig. 1A,B and Fig. 2 shows representative histological findings and morphometry at 6,15, 30 and 46 weeks in fa/fa and Fa/fa controls. Glomeruli of fa/fa rats appeared larger than those of $\mathrm{Fa} / \mathrm{fa}$ rats at each time point. As shown in Fig. 2A, by 6weeks (prior to development of overt diabetes or albuminuria) glomerular volume of fa/fa rats was already 1.6-fold greater than glomerular volume of $\mathrm{Fa} / \mathrm{fa}$ rats $(\mathrm{P}<0.01)$. The podocyte number per glomerulus in $\mathrm{fa} / \mathrm{fa}$ rat glomeruli was slightly but significantly higher than in control littermates at 6weeks $(\mathrm{P}=0.04)$ (Fig. 2B). Podocyte number per glomerulus decreased throughout the time course in $\mathrm{fa} / \mathrm{fa}$ rats with a slope of $-1.49 /$ week $(\mathrm{P}<0.01)$, but did not decrease significantly in $\mathrm{Fa} / \mathrm{fa}$ controls. Podocyte density (number per volume) in $\mathrm{fa} / \mathrm{fa}$ rats was decreased compared to $\mathrm{Fa} /$ fa controls by 6 weeks $(\mathrm{P}<0.01)$ and continued to decrease so that by 46 weeks the podocyte density had reached low levels of $50 \times 10^{6} / \mathrm{um}^{3}$ associated with development of glomerulosclerosis (Fig. $2 \mathrm{C}$ ). Glomerular volume per podocyte in $\mathrm{fa} / \mathrm{fa}$ rats was increased compared to $\mathrm{Fa} / \mathrm{fa}$ controls by 6 weeks $(\mathrm{P}<0.01)$ and continued to increase over the 46 week observation period (Fig. 2D). Total podocyte cell volume per glomerulus and average individual podocyte cell volume were already 1.5 -fold and 1.3 -fold respectively above control at 6 weeks $(\mathrm{P}<0.01)$ and became increasingly different from control over the 46 week observation period (Fig. $2 \mathrm{E}, \mathrm{F}$ ). In fa/fa rats by 30weeks PAS-positive expanded mesangial matrix was present (Fig. 1G) as also indicated by the increasing non-podocyte glomerular volume (Fig. 2G). By 46weeks expanded PAS-positive mesangial matrix was present in glomeruli (Fig. 1G) in association with segmental sclerosis lesions in $47 \pm 28 \%$ of glomeruli of fa/fa as assessed by Glepp1 peroxidase and AZAN staining (Fig. $1 \mathrm{H}$ and Supplemental Fig. 1A).

Relationship of morphometric parameters to albuminuria. $24 \mathrm{hr}$ urine albumin excretion was plotted against glomerular morphometric parameters (Fig. 3). There was a very strong relationship between podocyte nuclear density and $24 \mathrm{hr}$ albuminuria $\left(\mathrm{R}^{2}=0.86, \mathrm{P}<0.01\right)$ (Fig. $\left.3 \mathrm{~A}\right)$. Since podocyte density is the ratio of glomerular volume to podocyte number per glomerulus these parameters were separately plotted (Fig. 3B,C). The relationship of $24 \mathrm{hr}$ urine albumin to glomerular volume showed an $\mathrm{R}^{2}=0.70(\mathrm{P}<0.01)$. Similarly, there was a strong relationship between $24 \mathrm{hr}$ urine albumin and podocyte number per glomerulus $\left(\mathrm{R}^{2}=0.63, \mathrm{P}<0.01\right)$. Thus decreased podocyte density caused initially by increased glomerular volume alone, and subsequently by a combination of decreasing podocyte number compounded by increasing glomerular volume is strongly associated with albuminuria. However, fa/fa rat podocytes also appeared to be more susceptible than $\mathrm{Fa} / \mathrm{fa}$ podocytes to hypertrophic stress as shown by the higher level of albuminuria at a density of 100 podocytes $/ 10^{6} \mathrm{um}^{3}$ (Fig. 3A), implying that a podocyte susceptibility factor was also required for albuminuria to be present.

High growth milieu and podocyte hypertrophic stress. Fig. $4 \mathrm{~A}-\mathrm{C}$ show that at 6 weeks, prior to onset of glycosuria or albuminuria, blood insulin levels were 6.4-fold higher in $\mathrm{fa} / \mathrm{fa}$ rats than in $\mathrm{Fa} / \mathrm{fa}$ controls, and IGF1 and IGF2 (measured as $24 \mathrm{hr}$ urine excretion) were 3.2-fold and 2.1-fold increased respectively. These data reflect an increased growth milieu in fa/fa kidneys. At this time, glomerular volume and average podocyte volume 

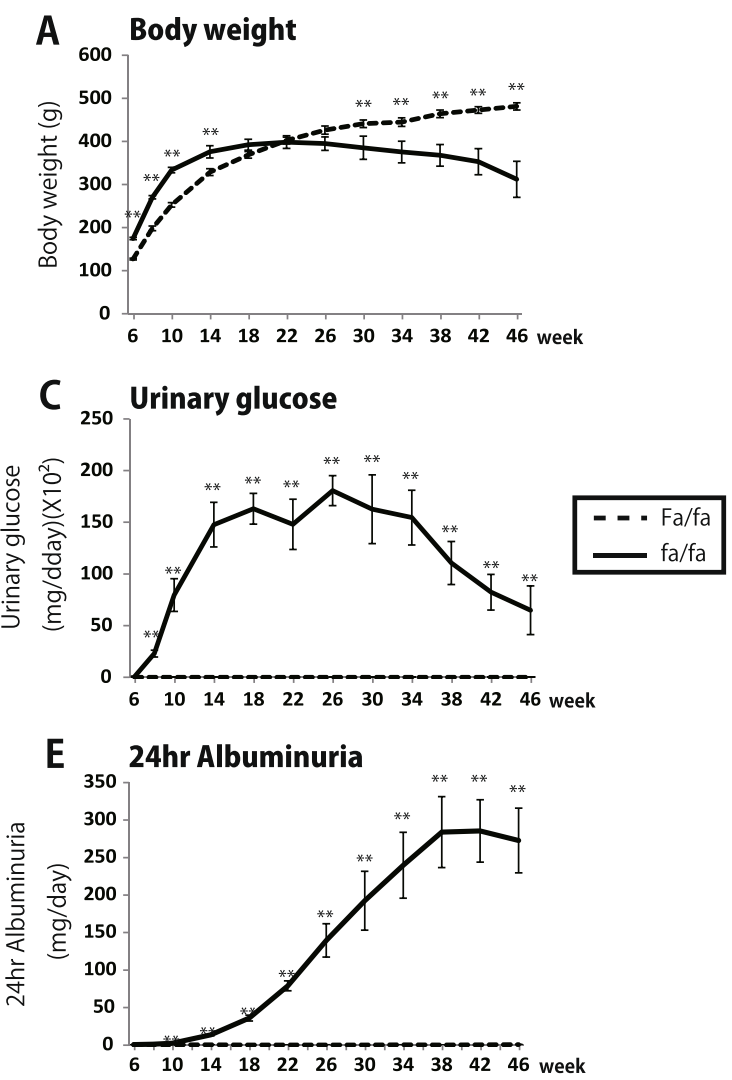
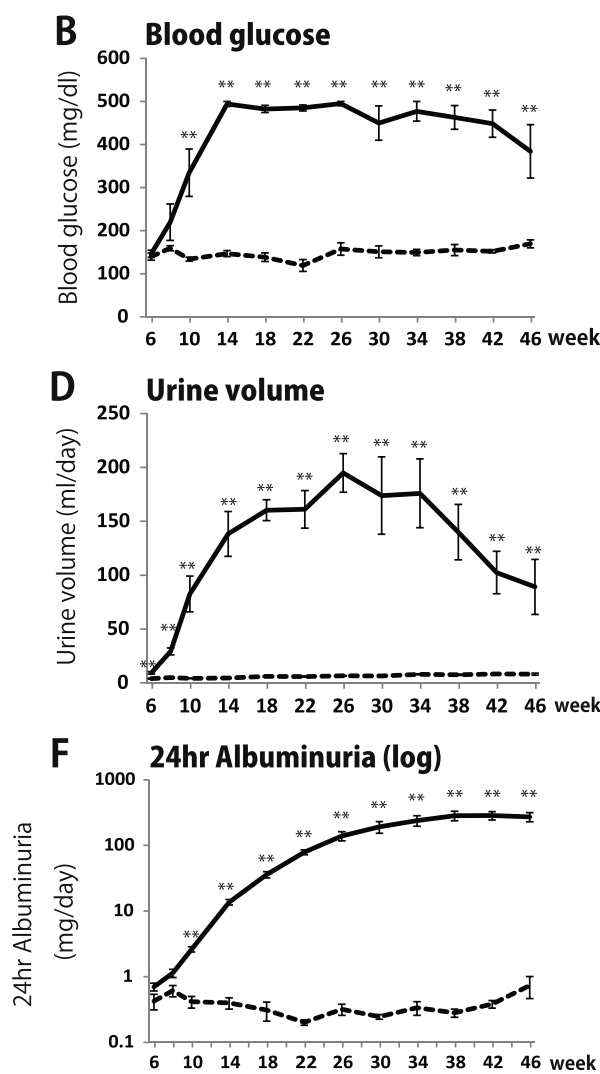

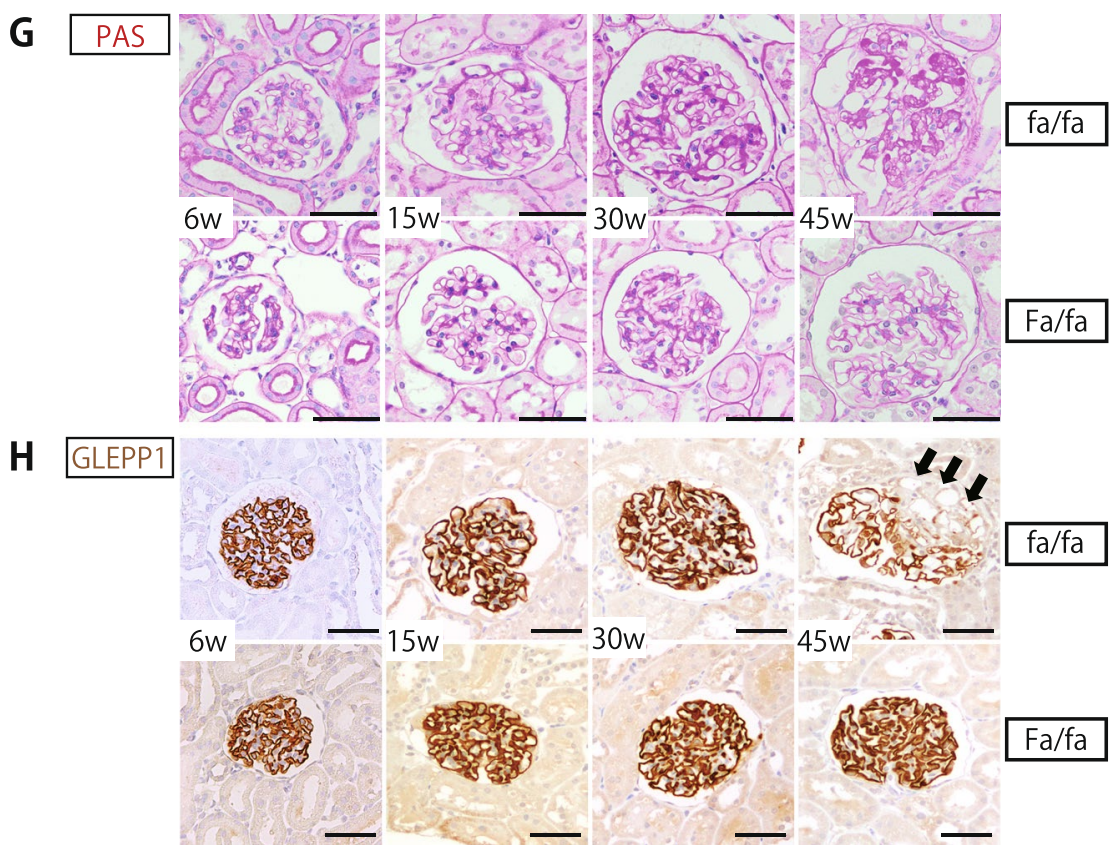

Figure 1. Time course of clinical parameters and histological figures in $\mathrm{fa} / \mathrm{fa}$ and $\mathrm{Fa} / \mathrm{fa}$ control rats. (A) Body weight gain. (B) Blood glucose. (C) $24 \mathrm{hr}$ urinary glucose. (D) $24 \mathrm{hr}$ urine volume. (E) $24 \mathrm{hr}$ urine albumin shown on a linear scale. (F) $24 \mathrm{hr}$ urine albumin shown on a log scale. (G) Representative PAS staining at 6 , 15,30 and 46 weeks of $\mathrm{fa} / \mathrm{fa}$ and $\mathrm{Fa} / \mathrm{fa}$ rats $(\mathrm{bar}=50 \mu \mathrm{m})$. $(\mathbf{H})$ Representative podocytes identified using the podocyte cytoplasmic marker glomerular epithelial protein 1 (GLEPP1) by immune-peroxidase $(\mathrm{bar}=50 \mu \mathrm{m})$. Glomeruli appear larger in $\mathrm{fa} / \mathrm{fa}$ rats than $\mathrm{Fa} /$ fa rats with increasing mesangial expansion over time in fa/fa rats shown by PAS staining. By 46 weeks segmentally sclerotic lesions were present in fa/fa rats affecting $47 \pm 28 \%$ of glomeruli, but were absent from glomeruli of $\mathrm{Fa} / \mathrm{fa}$ rats. Data shown as the mean $\pm \mathrm{SEM}$. Statistically significant differences are shown by asterisks $(* \mathrm{P}<0.05, * * \mathrm{P}<0.01)$. 

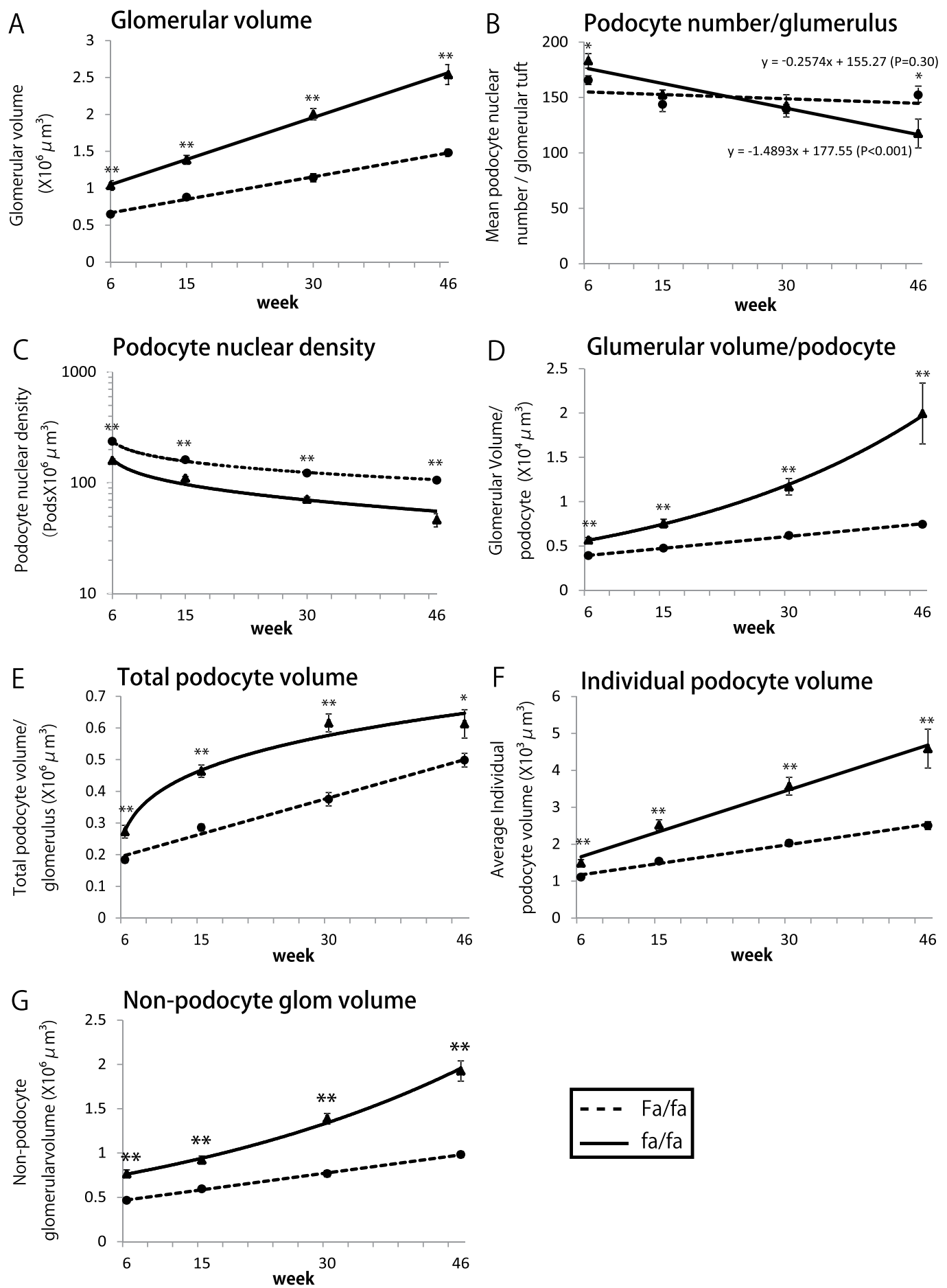

Figure 2. Morphometric parameters in fa/fa and fa/fa control rats. (A) Glomerular volume. (B) Podocyte nuclear number per glomerulus. (C) Podocyte nuclear density. (D) Glomerular volume per podocyte (E) Total podocyte volume per glomerulus (F) Average individual podocyte volume (G) Non-podocyte glomerular volume. By 6 weeks (pre-diabetic) glomerular volume in fa/fa rats was significantly increased above that of $\mathrm{Fa} / \mathrm{fa}$ rats $(\mathrm{P}<0.01)$. Over time podocyte number per glomerulus in $\mathrm{fa} / \mathrm{fa}$ rats decreased at a rate of -1.49 podocytes per week $(\mathrm{P}<0.01)$, while podocyte number per glomerulus did not change significantly over time in $\mathrm{Fa} / \mathrm{fa}$ rats $(\mathrm{P}=0.30)$. At 6 weeks the podocyte nuclear density (number density) was significantly lower in $\mathrm{fa} / \mathrm{fa}$ rats than $\mathrm{Fa} / \mathrm{fa}$ controls $(<0.01)$. To compensate for reduced podocyte density the average individual podocyte volume was increased 1.3 -fold in $\mathrm{fa} / \mathrm{fa}$ rats above control by 6 weeks $(\mathrm{P}<0.01)$. However, at 6 weeks total podocyte volume (podocyte number $\mathrm{x}$ size) had not fully adapted to glomerular volume increase as shown by the glomerular volume per podocyte. Statistically significant differences are shown by asterisks $(* \mathrm{P}<0.05$, $* * \mathrm{P}<0.01)$. 

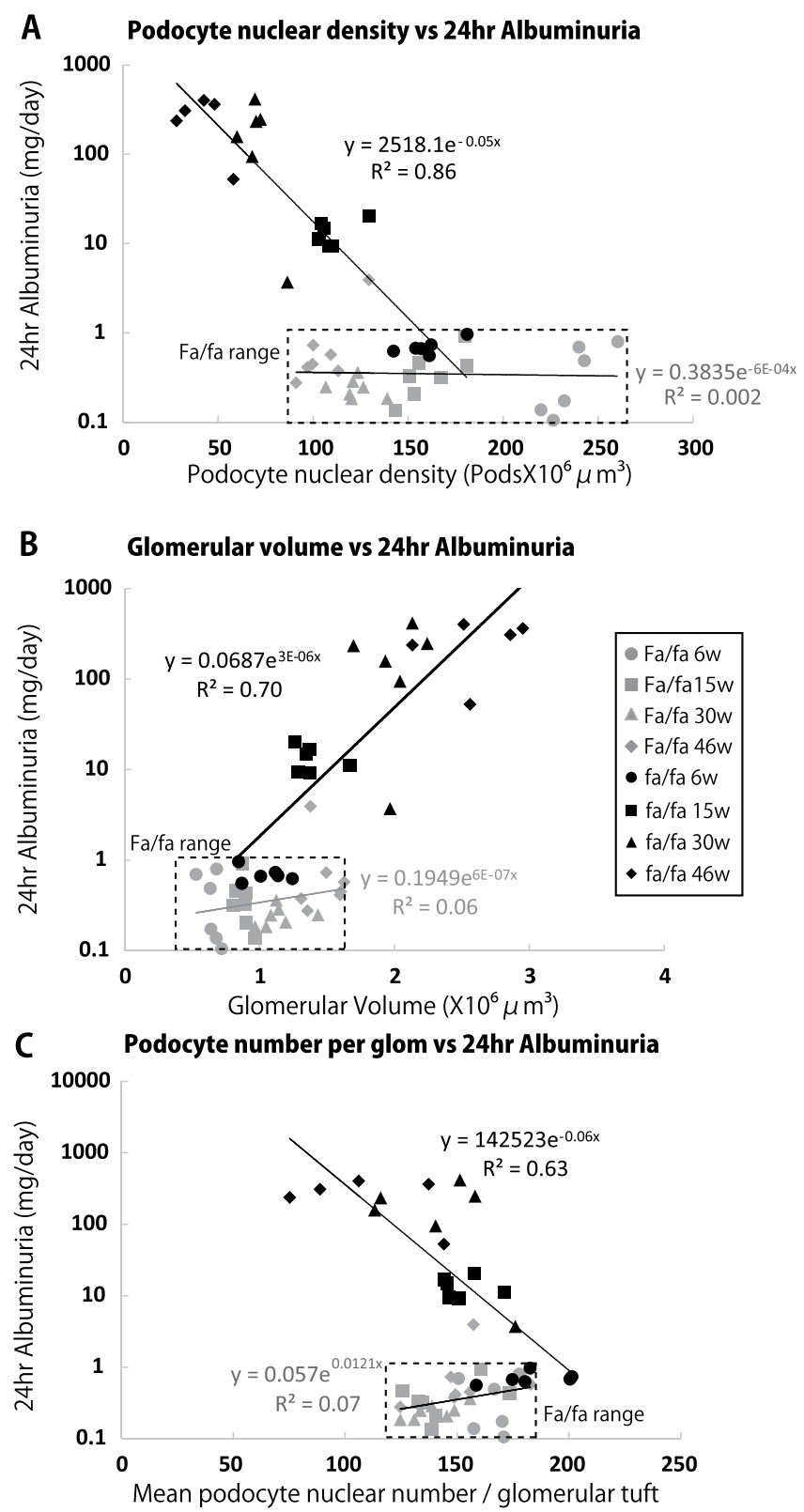

Figure 3. Relationship of morphometric parameters to $24 \mathrm{hr}$ urine albumin excretion. (A) Relationship between podocyte nuclear density and $24 \mathrm{hr}$ urine albumin excretion. (B) Relationship between glomerular volume and $24 \mathrm{hr}$ urine albumin excretion. (C) Relationship between podocyte nuclear number per glomerulus to $24 \mathrm{hr}$ urine albumin excretion. There is a very strong linear relationship between podocyte nuclear density and $24 \mathrm{hr}$ albuminuria for $\mathrm{fa} / \mathrm{fa}$ rats $\left(\mathrm{R}^{2}=0.86, \mathrm{P}<0.01\right)$ that was not present for $\mathrm{Fa} / \mathrm{fa}$ rats. The relationship of $24 \mathrm{hr}$ urine albumin to glomerular volume showed an $\mathrm{R}^{2}=0.70(\mathrm{P}<0.01)$. Similarly, there was a strong relationship between $24 \mathrm{hr}$ urine albumin and podocyte number per glomerulus $\left(\mathrm{R}^{2}=0.63, \mathrm{P}<0.01\right)$. Panel $\mathrm{A}$ shows that at a podocyte density of about 100 pods $/ 10^{6} \mathrm{um}^{3} \mathrm{fa} / \mathrm{fa}$ rats had a $24 \mathrm{hr}$ urine albumin of about $10 \mathrm{mg} /$ day. In contrast at the same podocyte density $\mathrm{Fa} / \mathrm{fa}$ rat $24 \mathrm{hr}$ urine albumin was $<1 \mathrm{mg} /$ day. Therefore $\mathrm{fa} / \mathrm{fa}$ rat podocytes had increased susceptibility to hypertrophic stress at the same podocyte density.

in fa/fa rats were already 1.6-fold and 1.3-fold above control respectively (Fig. 2A,F). Furthermore, the ratio of glomerular volume to total podocyte volume was higher in $\mathrm{fa} / \mathrm{fa}$ than in $\mathrm{Fa} / \mathrm{fa}$ controls $(\mathrm{P}<0.01)$ (Fig. 2D) demonstrating that podocyte hypertrophy had not kept pace with glomerular volume increase. Growth factors and nutrients drive cell growth via the mTORC1 pathway in part by phosphorylation of ribosomal protein S6. As shown in Fig. 4D,E, by 6weeks podocyte ribosomal S6 phosphorylation was already significantly increased in fa/ fa rat versus controls, compatible with hypertrophic podocyte stress being already present by 6 weeks.

Urinary mRNA markers of podocyte hypertrophic stress. Hypertrophic podocyte stress causes accelerated podocyte detachment which can be monitored non-invasively by measuring podocyte mRNAs in the 


\section{A Serum insulin}

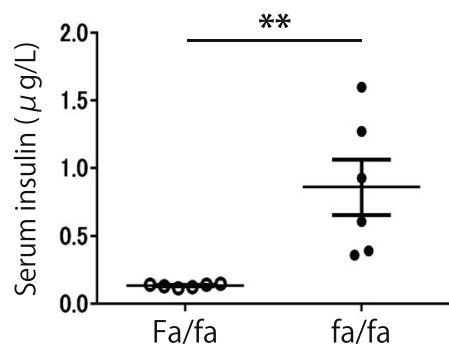

B

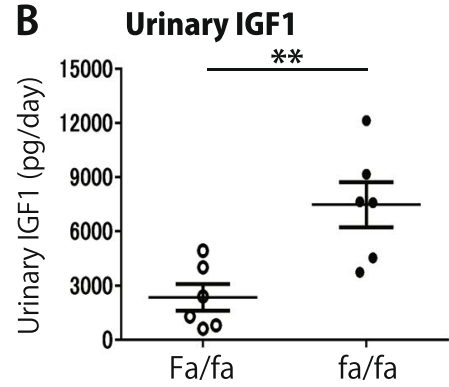

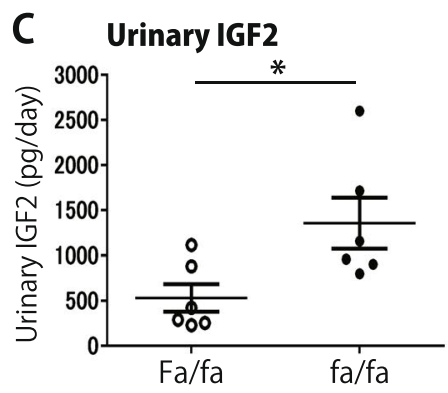

D

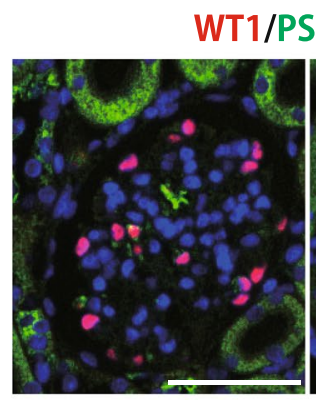

$\mathrm{Fa} / \mathrm{fa}$

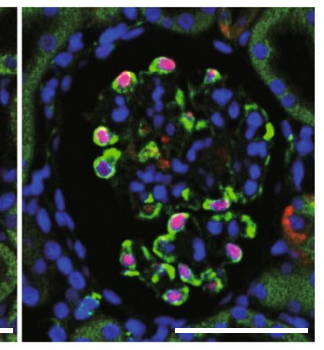

$\mathrm{fa} / \mathrm{fa}$
E

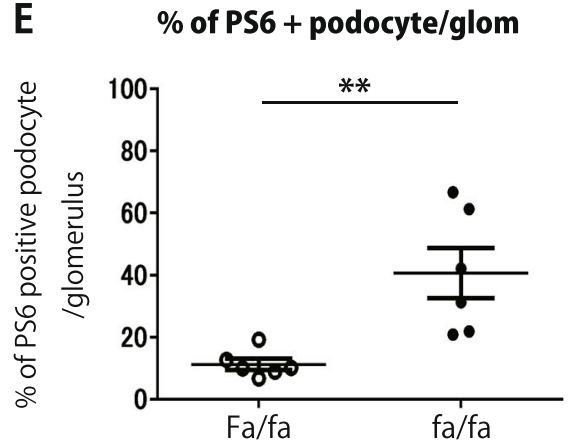

Figure 4. Pre-diabetic 6 week values for serum insulin, $24 \mathrm{hr}$ urine growth factor excretion and phosph-S6 immunostaining of $\mathrm{Fa} / \mathrm{fa}$ and fa/fa rats. (A) Serum insulin at 6 week. (B) $24 \mathrm{hr}$ urinary IGF1 at 6 week. (C) $24 \mathrm{hr}$ urinary IGF2 at 6week. (D) Immunofluorescent identification of phosphorylated ribosomal S6 (green cytoplasmic staining) at 6weeks prior to onset of overt hyperglycemia. Podocytes are shown by red WT1 nuclear staining. DAPI (blue) shows all nuclei. (E) Quantitation of glomerular ribosomal phosphor-S6 kinase in $\mathrm{fa} / \mathrm{fa}$ versus $\mathrm{Fa} / \mathrm{fa}$ rat glomeruli at 6 weeks. These data show the high growth factor milieu prior to onset of overt diabetes in $\mathrm{fa} / \mathrm{fa}$ rats compared to $\mathrm{Fa} /$ fa controls and increased ribosomal-S6 phosphorylation as a result of mTORC1 activation. $n=6$ for the Fa/fa group, $n=6$ for the fa/fa group, $* P<0.05$ and $* * P<0.01$, assessed using the Mann-Whitney U test.

urine pellet ${ }^{12,15}$. Figure 5A-D shows $24 \mathrm{hr}$ urinary pellet mRNAs for two podocyte markers (NPHS2/podocin and NPHS1/nephrin) and a distal tubular/collecting duct marker (aquaporin2). Also shown is the podocin:aquaporin 2 mRNA ratio as a measure of the relative rate of podocin mRNA excretion in relation to a tubular mRNA marker (aquaporin2). Figure $5 \mathrm{C}$ confirms that $24 \mathrm{hr}$ urinary pellet aquaporin $2 \mathrm{mRNA}$ levels were comparable between $\mathrm{fa} / \mathrm{fa}$ and $\mathrm{Fa} / \mathrm{fa}$ rats over the time-course in spite of large differences in urine volume between fa/fa and $\mathrm{Fa} /$ fa rats. By 6 weeks, prior to onset of hyperglycemia or albuminuria, both the urinary pellet podocin mRNA and podocin:aquaporin $2 \mathrm{mRNA}$ ratio in fa/fa rat were already significantly increased above that of controls $(\mathrm{P}=0.04)$, compatible with hypertrophic podocyte stress causing accelerated podocyte detachment at this early time point. Urinary podocin $\mathrm{mRNA}$ and podocin:aquaporin $2 \mathrm{mRNA}$ ratio subsequently increased reaching a plateau by 22 weeks and persisted throughout the 46 weeks of study at an average 88 -fold higher levels in fa/fa rat urine compared to $\mathrm{Fa} /$ fa rat controls over the period of observation. The relative level of two podocyte-specific mRNAs (podocin and nephrin) is a non-invasive measure of podocyte stress caused by relative down-regulation of nephrin versus podocin ${ }^{10,25}$. Figure 5E shows that by 8 weeks, prior to development of hyperglycemia or albuminuria, the urinary podocin:nephrin mRNA ratio was already significantly increased above control $(\mathrm{P}<0.01)$. Figure $5 \mathrm{~F}-\mathrm{J}$ show that the changes observed in the urinary pellet were also present in renal cortex at the 30 week time point.

The high nutrient and growth factor milieu in fa/fa rats might be expected to facilitate accelerated cell cycling as a part of the growth process. To assess this possibility, we used Ki67 as a cell cycle marker and WT1 and PAX8 as podocyte and parietal epithelial cell (PEC) markers respectively. As shown in Supplemental Table 1 although Ki67 labelling was present in glomeruli, interstitial and tubular compartments in both fa/fa and Fa/fa rats, there was no statistically significant difference between these groups. Furthermore, we were not able to identify double-label WT1/Ki67 positive cells (or double label PAX8/Ki67 PECs) in fa/fa rat kidney although double labelled cells were detectable in 2day old kidneys (Supplemental Fig. 2), suggesting that if de novo podocyte or PEC replenishment was occurring it was below the threshold of detection.

Food intake reduction (CR) "freezes" the progression process in place. If excess nutrient intake causes the altered kidney milieu that in turn causes podocyte hypertrophic stress and accelerated detachment leading to proteinuria and glomerulosclerolsis, then reducing nutrient intake should halt this process. To test this hypothesis rats were allowed to develop diabetes up to 15 weeks on an ad-libitum diet and were then placed on a $40 \%$ reduced food intake (CR) diet in comparison to an ad-libitum fed group. As shown in Fig. 6 and 


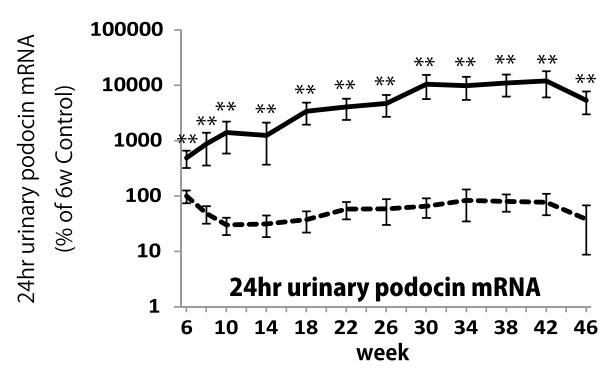

B

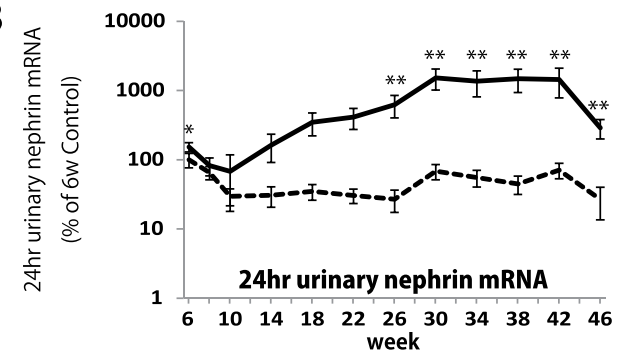

$\mathbf{F}$

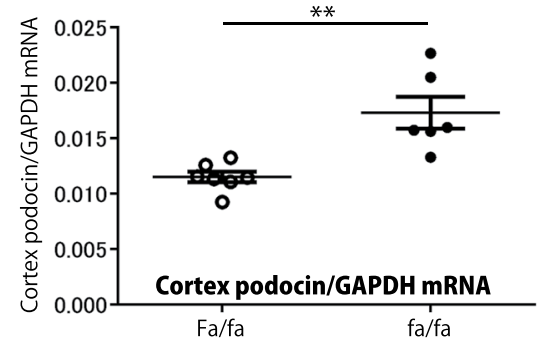

G

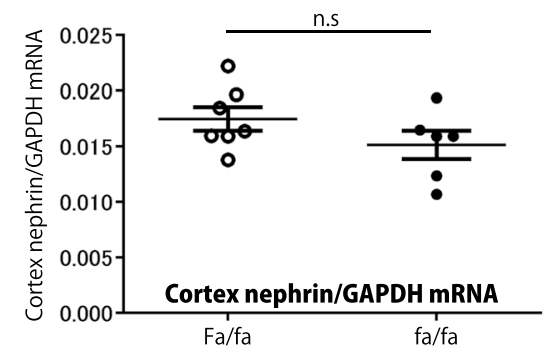

C

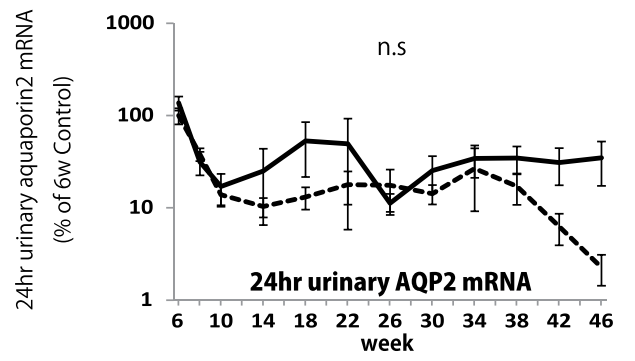

H

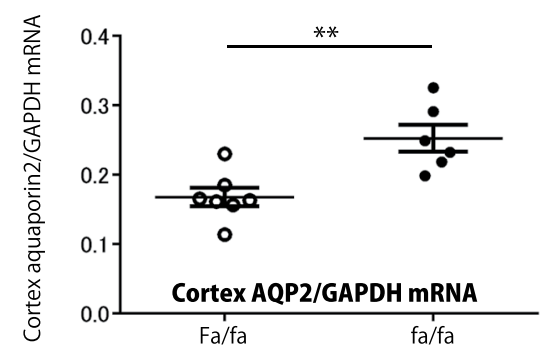

D
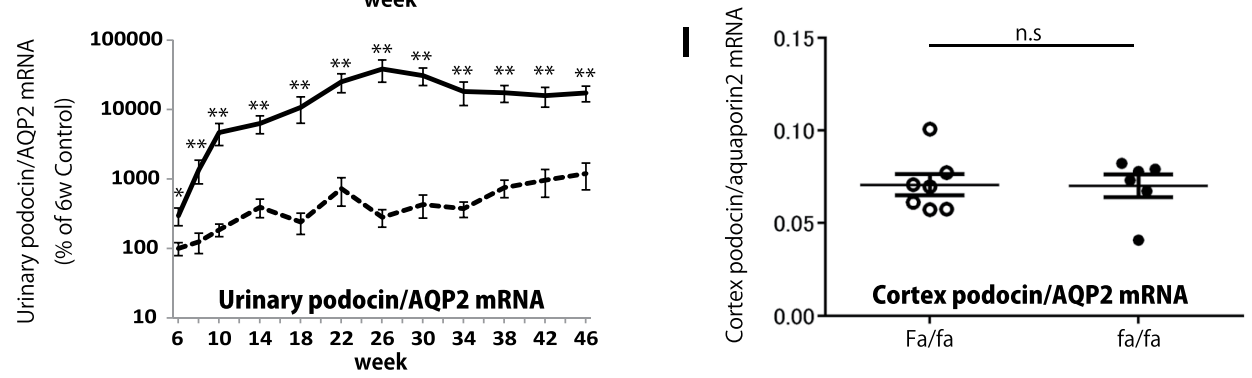

E
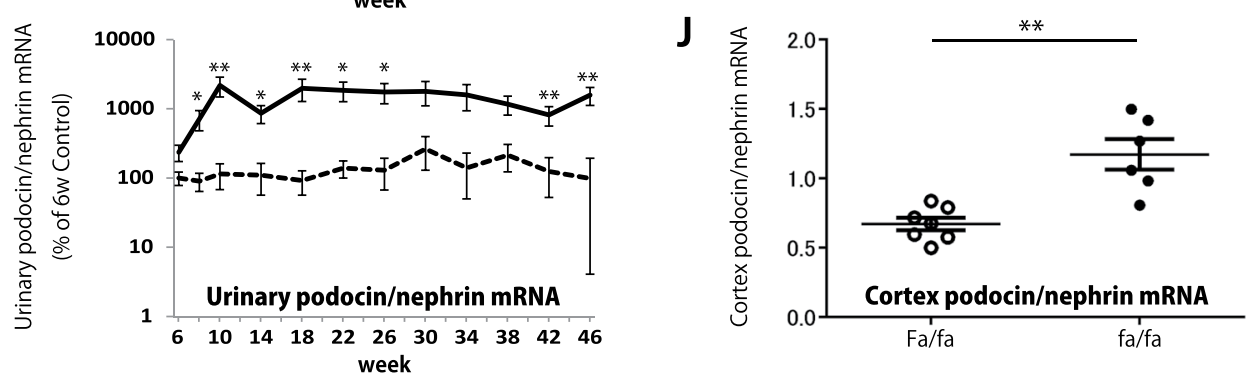

Figure 5. Time course of urinary pellet and kidney cortex mRNAs in fa/fa and Fa/fa control rats. (A) $24 \mathrm{hr}$ urinary podocin mRNA. (B) $24 \mathrm{hr}$ urinary nephrin mRNA. (C) $24 \mathrm{hr}$ urinary aquaporin $2 \mathrm{mRNA}$. (D) Urinary podocin:aquaporin 2 mRNA ratio per day. (E) Urinary podocin:nephrin mRNA ratio per day. (F) Kidney cortex podocin:GAPDH mRNA ratio at 30weeks. (G) Kidney cortex nephrin:GAPDH mRNA ratio at 30weeks. (H) Kidney cortex aquaporin 2:GAPDH mRNA ratio at 30weeks. (I) Kidney cortex podocin:aquaporin 2 mRNA ratio at 30weeks. (J) Kidney cortex podocin:nephrin mRNA ratio at 30weeks. By 6 weeks, prior to onset of overt diabetes or albuminuria, the urinary podocin mRNA excretion and the podocin:aquaporin $2 \mathrm{mRNA}$ ratio were already significantly increased above $\mathrm{Fa} / \mathrm{fa}$ control $(\mathrm{P}=0.04)$ and increased over the time course. The urinary podocin:nephrin mRNA ratio in the urine pellet as a measure of podocyte hypertrophic stress was also decreased by 8 weeks $(\mathrm{P}<0.05)$. For comparison at 30 weeks the renal cortical podocin:GAPDH mRNA ratio in $\mathrm{fa} /$ fa rats was 1.5 -fold increased $(\mathrm{P}<0.01)$ Statistically significant differences are shown by asterisks $(* \mathrm{P}<0.05$, $* * \mathrm{P}<0.01)$. 

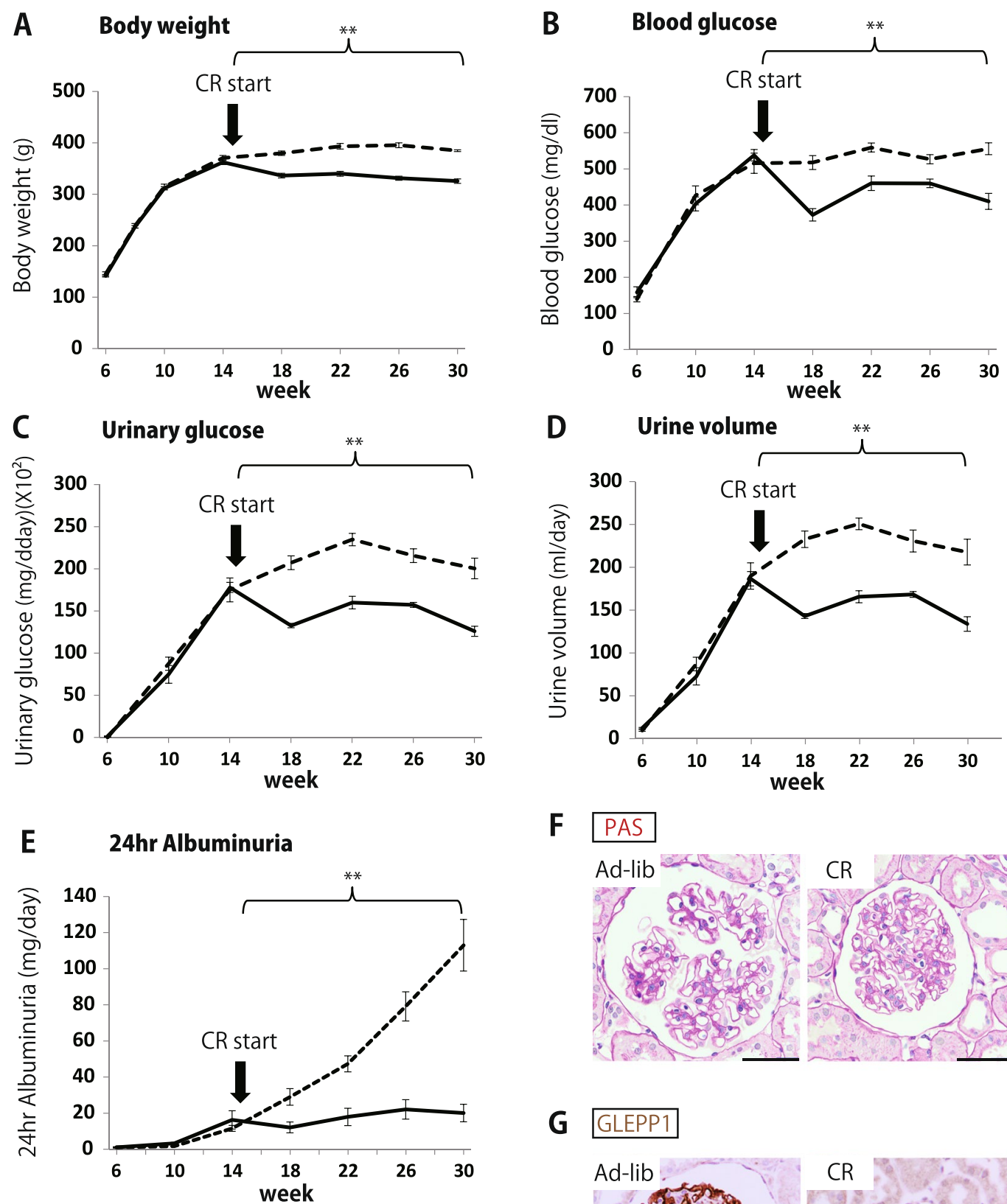

\section{F PAS}
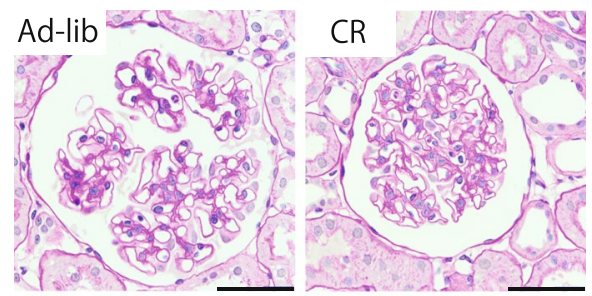

\section{G GLEPP1}
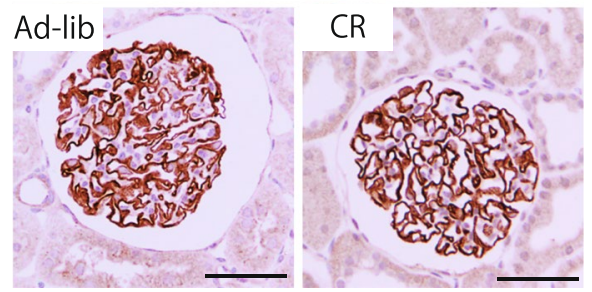

Figure 6. Time course of effect of switching to a calorie-reduced diet at 15 weeks and histological figures in the fa/fa rat. (A) Body weight. (B) Blood glucose. (C) $24 \mathrm{hr}$ urine glucose excretion. (D) Urine volume. (E) $24 \mathrm{hr}$ urinary albumin excretion. (F) Representative PAS staining at 30 weeks of ad-libitum and $40 \%$ calorie intake reduction $(\mathrm{CR})$ group in $\mathrm{fa} / \mathrm{fa}$ rats $(\mathrm{bar}=50 \mu \mathrm{m})$. $(\mathbf{G})$ Representative podocytes identified using the podocyte cytoplasmic marker glomerular epithelial protein 1 (GLEPP1) by immune-peroxidase at 30 weeks of ad-libitum and $40 \%$ calorie intake reduction $(\mathrm{CR})$ group in $\mathrm{fa} / \mathrm{fa}$ rats $(\mathrm{bar}=50 \mu \mathrm{m})$. The $40 \%$ reduced food intake group $(\mathrm{CR}$, solid line, $\mathrm{n}=6)$ gained less weight, had lower blood glucose, less glycosuria, lower urine volume and less albuminuria compared with the ad libitum diet group (dashed line, $n=5$ ). No histologic differences were detected between $\mathrm{CR}$ and ad libitum fed rats. ${ }^{*} \mathrm{P}<0.01$, assessed using the Mann-Whitney $\mathrm{U}$ test at the average 15-30week.

Supplemental Fig. 3, CR had the effect of stabilizing ("freezing") all parameters at the level they were at when CR was initiated at 15 weeks of age. Thus, although CR rats had statistically significantly less weight gain and lower blood glucose levels, hyperglycemia persisted at high levels over the 15weeks of observation (from week15 to 
week30). Ad libitum fed fa/fa rat $24 \mathrm{hr}$ urinary albumin excretion continued to increase to $100 \mathrm{mg} /$ day, while $\mathrm{CR}$ rats maintained stable albuminuria at about $20 \mathrm{mg} /$ day. Histology showed increased PAS-positive mesangial matrix in ad-libitum fed but not CR rats (Fig. 6F,G). Morphometry (Fig. 7A-E) showed that CR rats had lower glomerular volume $(\mathrm{P}=0.05)$, lower glomerular volume per podocyte and non-podocyte glomerular volume $(\mathrm{P}<0.01)$ and a higher podocyte nuclear density $(\mathrm{P}<0.05)$, although podocyte number per glomerulus was not significantly different $(\mathrm{P}=0.30)$. Thus $\mathrm{CR}$ rats at 30 weeks had similar morphometric parameters to the ad-libitum fed rats at 15weeks (see Fig. 2), consistent with CR slowing or halting growth-induced processes.

Figure $7 \mathrm{C}$ shows that the effect of CR was to stabilize podocyte density at a level of 82 podocytes $/ 10^{6} \mathrm{um}^{3}$ which, from Fig. 3A, would be predicted to correspond to a $24 \mathrm{hr}$ urinary albumin excretion of about $20 \mathrm{mg} /$ day similar to that observed in Fig. 6E. In contrast, the ad-libitum fed rat podocyte nuclear density decreased to $65 / 10^{6} \mathrm{um}^{3}$ primarily as a result of continued glomerular growth. From Fig. 3 A a podocyte density of $65 / 10^{6} \mathrm{um}^{3}$ is predicted to correspond to a $24 \mathrm{hr}$ urinary albumin excretion rate of close to $100 \mathrm{mg} /$ day as observed in Fig. $6 \mathrm{E}$. Thus the observed $24 \mathrm{hr}$ urinary albumin excretion values correspond closely to the values predicted by the podocyte density measurements thereby providing additional support for the concept that podocyte density is the major determinant of albuminuria.

Effect of CR on the growth milieu and podocyte hypertrophic stress. Fig. 7F-I shows that CR was associated with lower urinary IGF1/2 excretion. Furthermore, after 15weeks of CR glomerular ribosomal phosphor-S6 positive podocytes were reduced from $78 \%$ to $28 \%(\mathrm{P}<0.01)$ demonstrating that $\mathrm{CR}$ also reduced mTORC1-induced hypertrophic podocyte stress.

Reduced podocyte stress on the CR diet could be monitored by reduced urine podocyte mRNA markers. Figure 8 shows that CR was associated with a reduced rate of podocyte detachment as measured in both urinary pellet and in renal cortex $(\mathrm{P}<0.01)$. Although the cortical podocin:nephrin mRNA ratio was reduced by $\mathrm{CR}(\mathrm{P}<0.05)$, it was not reduced in the urinary pellet (Supplemental Fig. 3 ), suggesting that detaching podocytes were those that were stressed and had a high urinary podocin:nephrin mRNA ratio.

\section{Discussion}

Insulin and insulin-like growth factor (IGF-1/2) signaling (IIS pathway) in mammals, characteristically activated by nutrients and inhibited by starvation, is the paralogue of the insulin-like receptor daf- 2 in the nematode C. elegans that has provided remarkable insights into relationships between nutrition, growth and longevity ${ }^{26-28}$. IGF-1 under the influence of both growth hormone and local factors is a major driver of kidney growth ${ }^{29}$. Cell growth is coordinated through mTORC1 activation driven both by growth factors binding to cell surface receptors and nutrient sensing of amino acids and glucose $\mathrm{e}^{30,31}$. Hyperphagic fa/fa rats with secondary hyperinsulinemia, high IGF1/2, and large nutrient intake are therefore predisposed to activate mTORC1 and thereby to grow rapidly, including rapid glomerular growth. At the same time the podocyte is a structurally complex cell with limited capacity to divide or hypertrophy rapidly. Furthermore, primary dysregulation of the mTORC1 pathway in podocytes is proven to cause thickened GBM, proteinuria and other diabetes-like pathologic changes in the absence of hyperglycemia ${ }^{12,15,20,21,32}$. Thus, in fa/fa rats a high growth milieu can drive both glomerular volume enlargement and podocyte mTORC1 activation. In this setting the stressor (glomerular enlargement) and the susceptibility factor (podocyte mTORC1 activation) combine to cause accelerated podocyte detachment, albuminuria, glomerulosclerosis, and ultimately progression to ESKD, as previously reported for a non-diabetic model ${ }^{12,15}$.

Kriz and Lemley have previously emphasized that podocyte loss from glomeruli occurs primarily by detachment $^{33}$. We therefore evaluated whether podocyte mRNA markers detected in the urinary pellet could quantitatively account for the number of podocytes lost from fa/fa rat glomeruli over the 40week period of observation. As shown in Supplemental Table 2, we estimate that $>70 \%$ of podocytes lost from glomeruli could in fact be accounted for in the urinary pellet. Non-invasive markers that quantitatively reflect podocyte stress prior to hyperglycemia or albuminuria onset and that non-invasively report the rate of podocyte detachment from glomeruli have potentially clinical utility. We have previously reported similar associations in other models ${ }^{10-12,15}$ as well as in human Alport syndrome ${ }^{14,34}$, IgA nephropathy ${ }^{35}$, anti-GBM disease ${ }^{16}$ and allograft failure ${ }^{36}$. Other investigators have reported similar findings ${ }^{37-42}$.

By 8 -10weeks in homozygous fa/fa rats overt diabetes reflected by hyperglycemia, glycosuria and polyuria was present. Increased albumin excretion was also present by 10 weeks, and reached high levels by 38 weeks. At first sight albuminuria might seem attributable to hyperglycemia itself, however, decreasing podocyte density is an alternative explanation for the progressive increase in albuminuria. This is an important distinction because if proteinuria and progressive glomerulosclerosis are significantly caused by glomerular volume enlargement this would identify an under-recognized therapeutic target for preventing progression. If glomerular enlargement causing podocyte hypertrophic stress is indeed a major driver of albuminuria in the fa/fa rat then one would expect that there would be a direct relationship between the $24 \mathrm{hr}$ urinary albumin excretion and glomerular volume itself, as was observed $\left(\mathrm{R}^{2}=0.70, \mathrm{P}<0.001\right)$. Furthermore, if the effect of glomerular volume enlargement in causing albuminuria was through podocyte hypertrophic stress then one would expect that podocyte density (glomerular volume in relation to podocyte number) would show an even stronger relationship with albuminuria than did glomerular volume alone, as was observed $\left(\mathrm{R}^{2}=0.86, \mathrm{P}<0.001\right)$. Glomerulosclerosis was present in $\mathrm{fa} /$ fa rat glomeruli by 46 weeks when podocyte density had reached low levels $\left(50 / 10^{6} \mathrm{um}^{3}\right)$ as a result of the combination of glomerular volume enlargement and podocyte depletion. Podocyte density at this level is associated with glomerulosclerosis in all models and human glomerular diseases so far examined ${ }^{12-19}$. These data are therefore compatible with prior work showing that glomerular volume increase per se in the setting of podocyte susceptibility to hypertrophic stress drives albuminuria and glomerulosclerosis ${ }^{12-16}$. 
A

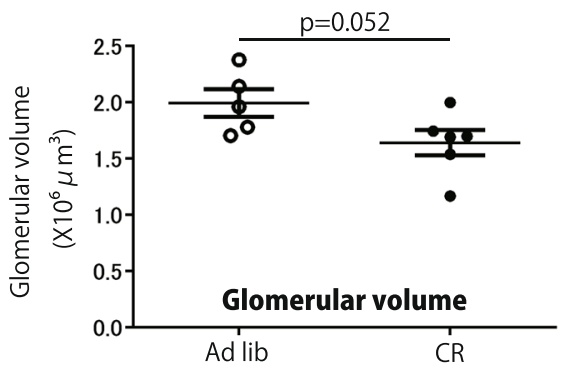

B

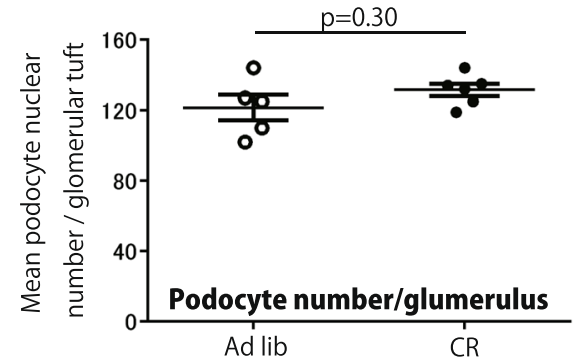

C

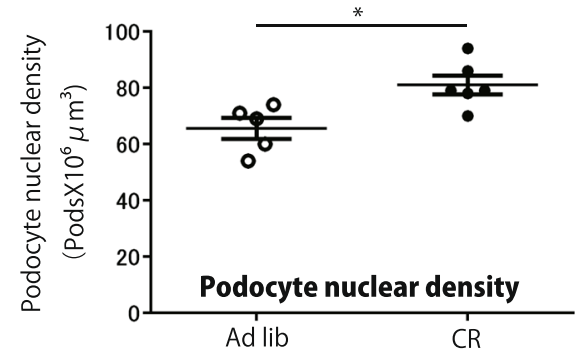

D

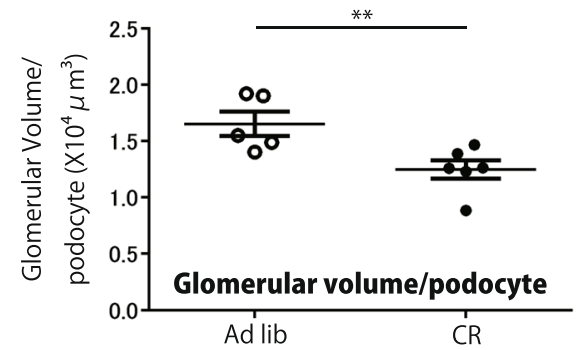

E

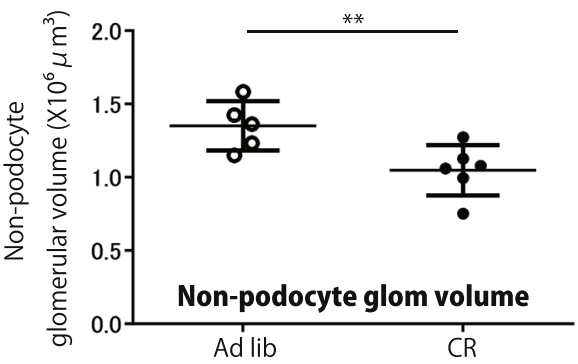

F Urinary IGF1

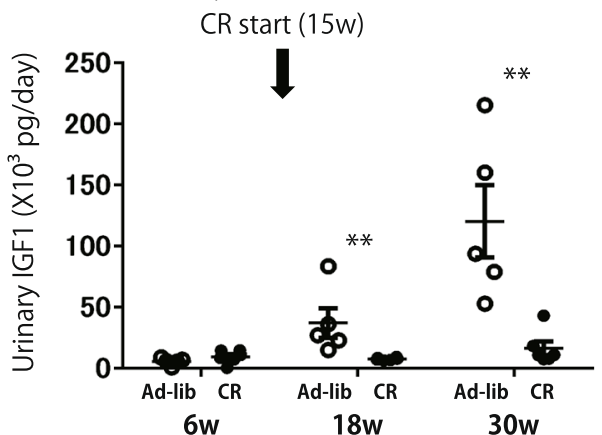

G Urinary IGF2

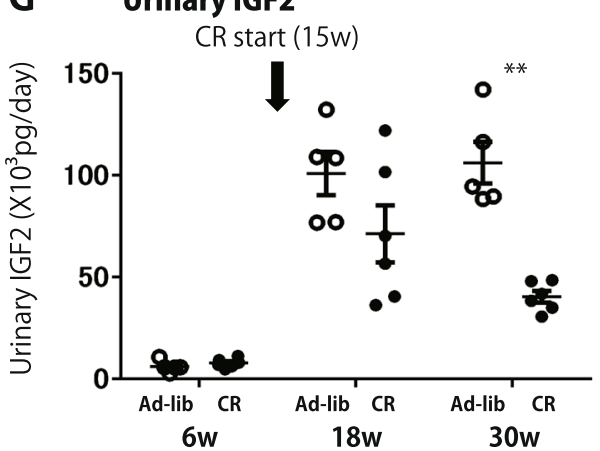

H

WT1/PS6/DAPI

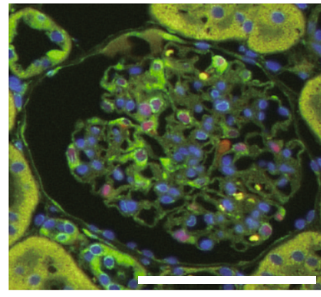

Ad lib

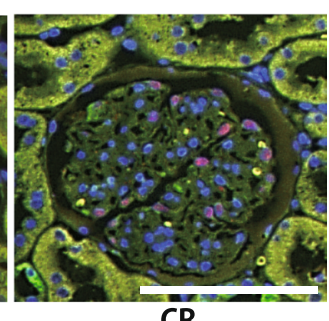

$30 w$

I $\%$ of PS6 + podocyte/glom

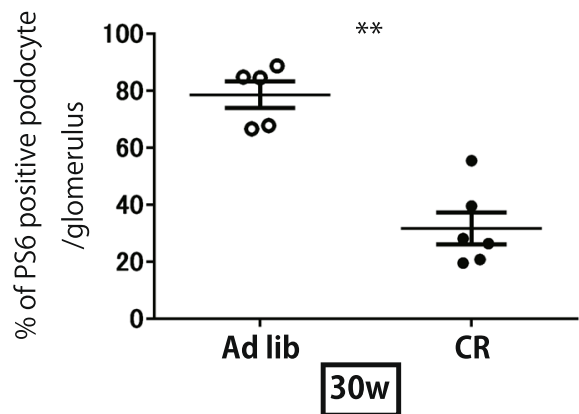

Figure 7. Morphometric analysis and effect on reduced calorie intake ( $40 \% \mathrm{CR})$ values for $24 \mathrm{hr}$ urine growth factor excretion and phosph-S6 immunostaining of fa/fa rats. (A) Glomerular volume. (B) Mean podocyte nuclear number per glomerular tuft. (C) Podocyte nuclear density. (D) Glomerular volume per podocyte. (E) Non-podocyte glomerular volume. (F) Effect of $40 \%$ CR on $24 \mathrm{hr}$ urinary IGF1. (G) Effect of $40 \%$ CR on $24 \mathrm{hr}$ urinary IGF2. (H) Representative immune-histologic findings at 30 weeks of ad-libitum and $40 \% \mathrm{CR}$ fa/fa rats. Phosphorylation of ribosomal S6 in glomeruli (green immunofluorescence). Podocyte nuclei are shown by red WT1 immunofluorescent staining. DAPI is shown in blue. (bar $=100 \mu \mathrm{m}$ ). (I) Quantification of $\%$ of ribosomal phosphor-S6 positive podocytes per glomerulus. The CR rats had less increase in glomerular volume although podocyte number per glomerulus did not change significantly, resulting in lower podocyte density reduction in CR rats than occurred in the ad libitum-fed group. CR was associated with lower urinary IGF1/2 excretion and reduced mTORC1-induced hypertrophic podocyte stress. $\mathrm{n}=5$ for ad-libitum diet group, $\mathrm{n}=6$ for $40 \% \mathrm{CR}$ group, $* \mathrm{P}<0.05$ and $* * \mathrm{P}<0.01$, assessed using the Mann-Whitney $\mathrm{U}$ test. 

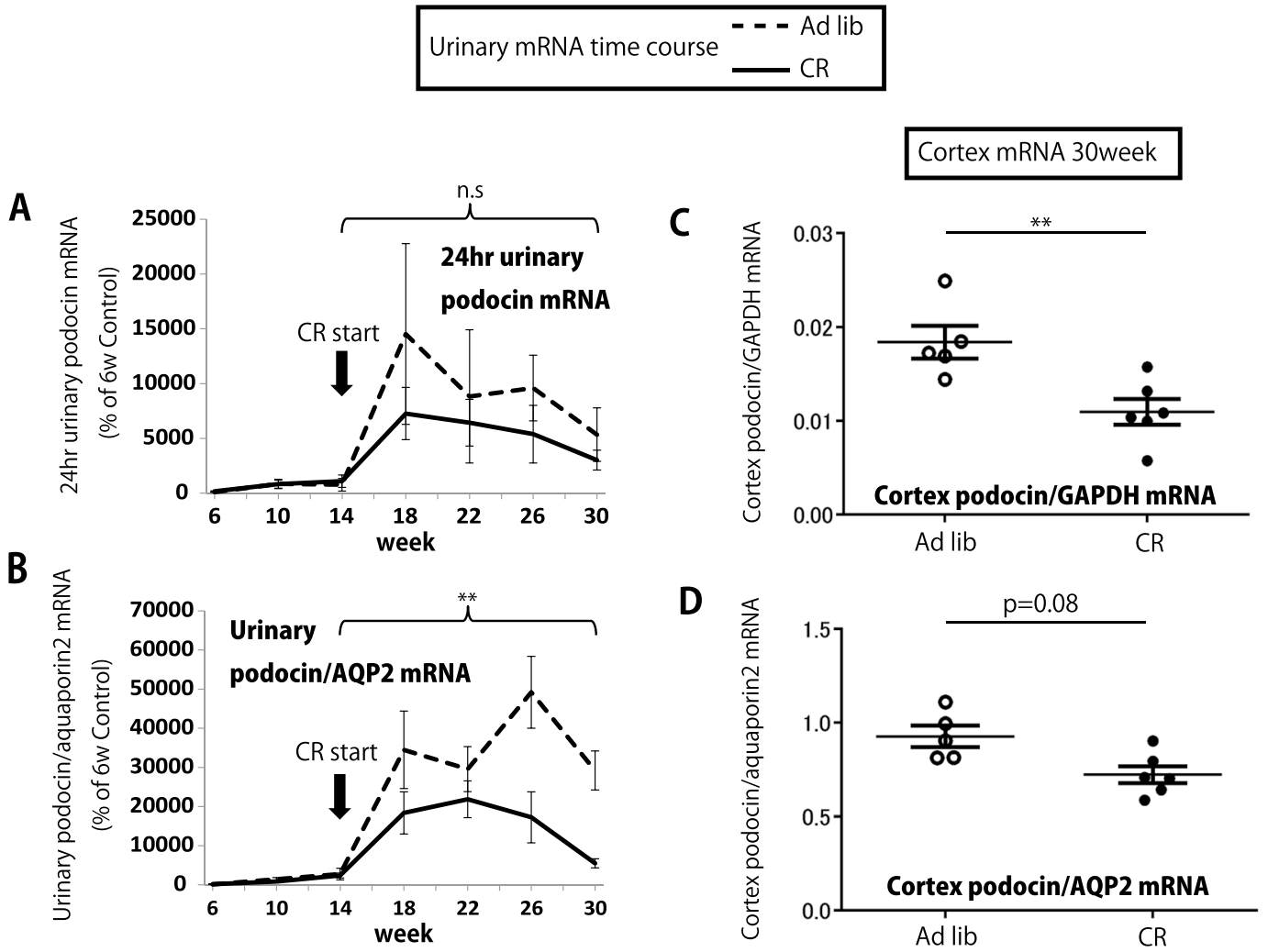

Figure 8. Urinary and kidney cortex mRNA in ad-libitum and calorie intake reduction (CR) groups in fa/fa rats. (A) Time course of $24 \mathrm{hr}$ urinary podocin mRNA. (B) Time course of urinary podocin:aquaporin $2 \mathrm{mRNA}$ ratio. (C) Kidney cortex podocin:GAPDH mRNA ratio at 30weeks. (D) Kidney cortex podocin:aquaporin 2 mRNA ratio at 30weeks. CR was associated with lower urinary pellet and renal cortical podocyte mRNA. $n=5$ for ad-libitum diet group, $\mathrm{n}=6$ for $40 \%$ calorie intake reduction group, $* \mathrm{P}<0.05$ and $* * \mathrm{P}<0.01$, assessed using the Mann-Whitney $U$ test using the average value of urinary data over the $15-30$ week time period.

Further support for this concept is provided by the food reduction (CR) study initiated at 15weeks after hyperglycemia and albuminuria were already established. In spite of persistent hyperglycemia, CR essentially "froze" the progression process in place such that all parameters including urinary IGF1/2, glomerular enlargement, podocyte density reduction, podocyte mTORC1 activation (ribosomal phosphor-S6), podocyte detachment rate, urinary podocyte markers and albuminuria remained at the stage they were at when CR was initiated. This suggests that podocytes were able to adapt to a lower stress level over the 15weeks of CR in spite of persistent hyperglycemia. Therefore, in the fa/fa rat hyperglycemia per se did not appear to be either necessary to initiate progression nor sufficient to drive the progression process once established.

Translation of $\mathrm{fa} / \mathrm{fa}$ rat findings to man has important caveats. First, is the fa/fa rat a model of human obesity-associated nephropathy or type 2 DAN or both? Obesity in the absence of overt diabetes is well-known to be associated with glomerular enlargement, proteinuria and development of FSGS lesions ${ }^{43}$. Furthermore, in this setting reducing food intake through bariatric surgery and other approaches ameliorates proteinuria ${ }^{44}$. On the other hand, type2 DAN also results from excessive food intake causing weight gain/obesity and hyperinsulinemia with superimposition of insulin resistance sufficient to cause pancreatic islet beta cell failure resulting in overt diabetes, as also occurs in the fa/fa rat model. Therefore, the fa/fa rat also resembles type2 DAN, particularly with respect to childhood type 2 diabetes ${ }^{45}$. A second related question is that although the fa/fa rat replicates morphologic characteristics found in association with type2 DAN including basement membrane thickening, PAS-positive mesangial expansion and high level proteinuria ${ }^{22}$, like most but not all other rodent models, it does not exactly replicate major nodular glomerulosclerosis considered to be a characteristic pathologic phenotype of $\mathrm{DAN}^{23,24}$. However, in reality, there is a wide range of pathology observed in biopsies performed on proteinuric diabetics with the proportion of biopsies showing non-classical diabetic features ranging from $14-83 \%{ }^{46}$ with $22 \%$ having FSGS ${ }^{47}$. The data in this report taken together with previous morphometric studies using a model of growth-induced glomerular failure ${ }^{12,15}$ and model of both type 1 and type 2 DAN in $\operatorname{man}^{17-19,48-50}$, would be consistent with an underlying process initiated by a combination of glomerular enlargement with podocyte susceptibilities. Podocyte susceptibilities could result from the growth factor milieu triggering podocyte mTORC1 activation, hyperglycemia level and exposure time (Fig. 9), metabolic factors including reactive oxygen species, mutations in podocyte-expressed genes such as the Col4 $\alpha 3$ gene recently reported to be associated with MODY ${ }^{51}$, superimposed hypertension and other factors. According to this perspective the $\mathrm{fa} / \mathrm{fa}$ rat models key elements common to both obesity and type 2 diabetes-associated nephropathies. Importantly, these events can be monitored non-invasively using urine pellet podocyte mRNAs from an early stage prior to onset of hyperglycemia or albuminuria. 


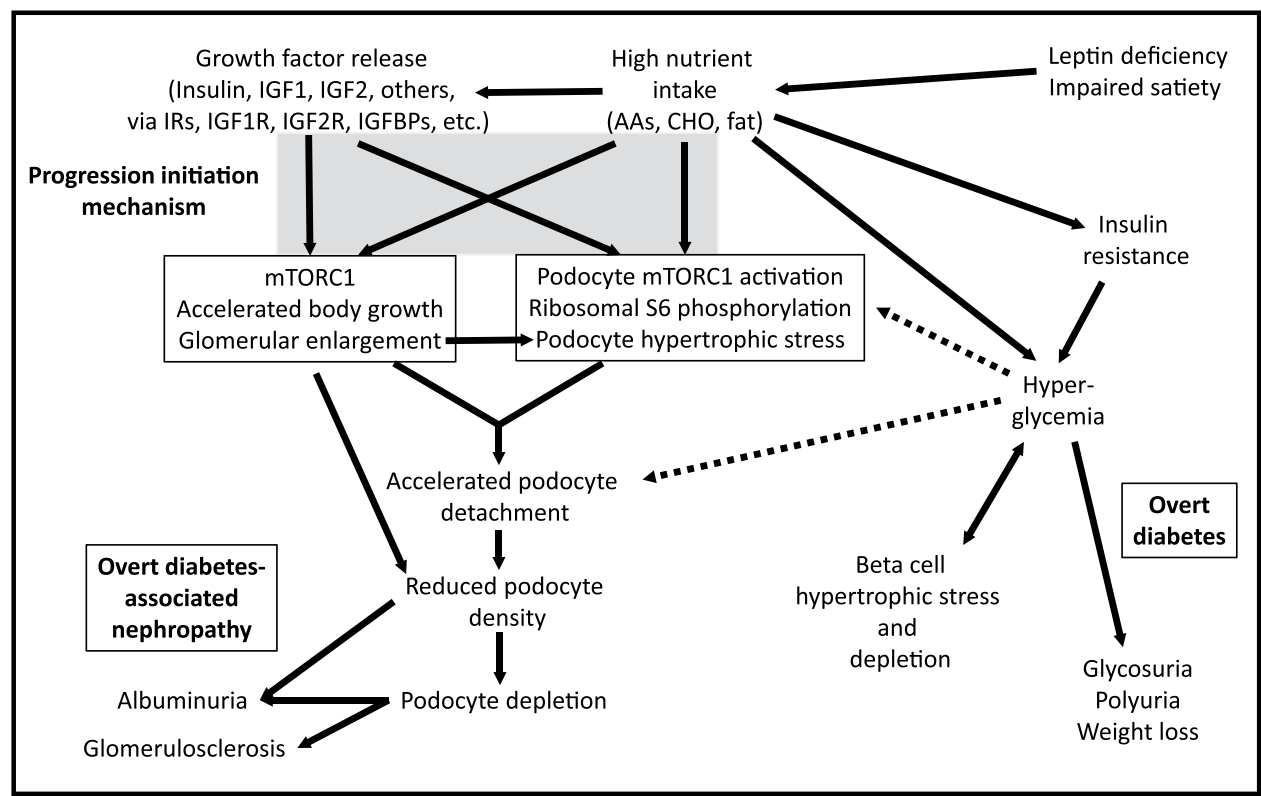

Figure 9. Schematic hypothesis for growth-induced progression derived from the fa/fa model. Nutrition-induced insulin and insulin-like growth factors (IGF-1/2) activate their individual receptors (insulin receptors [IRA and IRB], IGF-1R and IGF-2R, and their hybrid receptors comprising combinations of IR and IGF-1R proteins ${ }^{53}$. IGF-1 availability and local function is regulated through 6 different IGF1 binding proteins (IGFBPs1-6). Cell growth occurs through GF-induced activation of the mTORC1 complex which simultaneously senses nutrient availability including amino acids (leucine, arginine) and glucose ${ }^{30,31,54}$. In fa/fa rats high level nutrient intake drives high growth factor expression from birth resulting accelerated body and organ growth. IGF-1 promotes both glomerular volume enlargement and hyperfiltration ${ }^{29,55-57}$. Glomerular enlargement requires podocytes to hypertrophy to cover the expanding filtration surface with foot processes through increased protein synthesis via mTORC1 kinase-induced phosphorylation/activation of S6 kinase which in turn phosphorylates ribosomal S6. Thus, the collaboration between growth factors, nutrients, glomerular volume and podocytes represents the core components of a "progression initiation mechanism" (see the shaded box). Podocyte hypertrophic stress is represented in this report by the triad of high level podocyte ribosomal S6 phosphorylation, relative downregulation of the podocyte-specific transcript nephrin versus podocin, and accelerated podocyte detachment into the filtrate. Glomerular volume increase and accelerated podocyte detachment both drive reduced podocyte density. The level of reduced podocyte density in concert with podocyte stress determines amount of albumin leak through the filter, and thereby degree of albuminuria. Mesangial expansion reduces the filtration area required for foot process coverage to preserve filter integrity, but when podocyte density reduction reaches critically low values fibrosis supervenes at sites of podocyte depletion. In parallel, pancreatic islet beta cells hypertrophy to increase insulin release to adapt to increasing insulin resistance, but eventually fail and become depleted in association with loss of blood glucose control. This in turn leads to the cardinal features of diabetes mellitus (polyuria, polydipsia and weight loss). Thus, parallel failure of two structurally and functionally complex longlived cell types with limited capacity for replacement (pancreatic beta cells and podocytes) represent the diabetesassociated nephropathy phenotype. Hyperglycemia itself through oxidant injury or other mechanisms could also potentially contribute to podocyte detachment through various signaling pathways, mTORC1 complex activation via glucose sensors, or direct glucose toxicity effects ${ }^{7}$. However, in the fa/fa rat hyperglycemia itself did not appear to be required either to initiate podocyte injury or to sustain the progression mechanism once it was established. Hence the interaction between hyperglycemia and podocyte loss is represented by dashed lines in the schematic.

\section{Materials and Methods}

The study was approved by the University of Miyazaki Animal Research Committee (approval numbers: 2011-539 and 2017-501). Authors confirm that all the experiments are performed in accordance with approved guidelines.

Rat model of diabetic nephropathy. Male leptin-deficient Zucker (fa/fa) rats that develop type2 diabetes $(n=6)$ were compared with heterozygous $\mathrm{Fa} / \mathrm{fa}$ rat littermates $(\mathrm{n}=7)$ as non-diabetic controls. Rats were purchased from Charles River Breeding, Yokohama, Japan at 5weeks old. Open kidney wedge biopsy was performed with removal of about 1/10th of the kidney at 15 and 30 weeks. Kidneys were harvested at 46weeks at euthanasia when kidneys were perfusion-fixed with paraformaldehyde/lysine/periodate (PLP) and paraffin-embedded prior to sectioning.

Food intake reduction study (calorie reduction, CR). 5 weeks old male fa/fa rats ( $150 \mathrm{~g})$ were fed ad-libitum until 15 weeks of age when they were randomly assigned to either: (i) ad-libitum diet $(n=5)$, or (ii) $40 \% \mathrm{CR}(\mathrm{n}=6)$. Food delivered to the $40 \% \mathrm{CR}$ group was calculated by measuring the food eaten by the ad-libitum group at the same age. Rats were euthanized at 30 weeks when kidneys were perfusion-fixed for analysis. 
RNA analysis and qRT-PCR assay. Rats were housed in metabolic cages for overnight timed urine collection (average $17 \mathrm{hr}$ ) every 4weeks to measure urine volume, albuminuria, urinary glucose and urinary podocyte mRNA markers (podocin and nephrin) and aquaporin 2 mRNA excretion. Urine was centrifuged at $4{ }^{\circ} \mathrm{C}$ for $15 \mathrm{~min}$ at $3200 \times \mathrm{g}$ on a tabletop centrifuge. The supernatant was removed, the pellet suspended in $1.5 \mathrm{~mL}$ diethyl pyrocarbonate-treated phosphate-buffered saline, and then centrifuged at $12,000 \times g$ for $5 \mathrm{~min}$ at $4{ }^{\circ} \mathrm{C}$. Diced rat cortex from kidney biopsy samples and washed urine pellets were suspended in RLT/ $\beta$-mercaptoethanol buffer (RNeasy kit; Qiagen, Germantown, MD, USA) and then frozen at $-80^{\circ} \mathrm{C}^{11,16}$. RNA from the urinary pellet and kidney cortex were purified using an RNeasy mini kit (cat. No. 74106; Qiagen). cDNAs were reverse-transcribed from total RNA $(\sim 1 \mu \mathrm{g})$ using a high-capacity cDNA reverse transcription kit (Applied Biosystems, Foster City, CA, USA). Quantitation of podocin, nephrin, aquaporin2 and glyceraldehyde-3-phospate dehydrogenase (GAPDH) mRNA abundance was performed with a LightCycler $96^{\circledR}$ system (Roche Molecular System, Mannheim, Germany) using FastStart Essential DNA Probe Master Mix (Roche Molecular System, Inc.) in a final volume of $10 \mu \mathrm{L}$ per reaction. TaqMan probes (Applied Biosystems) used were: rat NPHS1 (nephrin) (cat. No. Rn00575235_m1); rat NPHS2 (podocin) (cat. No. Rn00709834_m1); rat aquaporin 2 (cat. No. Rn00563755_m1); rat GAPDH (cat. No. Rn01775763_m1). cDNA standard curves were constructed using these serially-diluted standards ${ }^{11,16}$. For analysis all urine measurements were adjusted to 24 hours.

Podometric analysis and phosphor-S6 immunostaining. Immunofluorescence and immunoperoxidase staining used PLP-perfused paraffin-embedded kidney sections. Mean glomerular radius (r) for 30-50 consecutive glomerular profiles was measured moving systematically from outer cortex to juxtamedullary glomeruli using the WinROOF imaging software (Mitani Corp., Tokyo, Japan). Mean maximal $\mathrm{R}=4 / \pi \mathrm{r}$ and mean glomerular volume $=4 / 3 \pi \mathrm{R}^{3}$ were calculated ${ }^{16,52}$. Podocyte nuclear number was measured using Wilms' Tumor 1 (WT1) immunofluorescence staining using 1.5um thick sections by ImagePro software (Media Cybernetics, Rockville, MD, USA) in 30-50 glomerular tufts ${ }^{52}$. Podocyte nuclear density was estimated using a quadratic equation developed to correct for section thickness and nuclear shape $e^{52}$. Average podocyte nuclear number per glomerular tuft = average glomerular volume x podocyte nuclear density. GLEPP1-positive tuft area was estimated from 30-50 consecutive glomerular tuft areas as the \%glomerular tuft area staining positive by GLEPP1 immunoperoxidase using the WinROOF imaging software ${ }^{16,52}$. Average total podocyte volume was estimated by multiplying the mean glomerular volume by the mean \%GLEPP1-positive area. The non-podocyte glomerular volume was estimated by subtracting total GLEPP1 podocyte volume from glomerular volume. Reagents: WT1 primary antibody (SC-7385 monoclonal IgG1; Santa Cruz Biotechnology, USA) 1:50 with Cy3-labeled secondary antibody (Jackson ImmunoResearch Laboratories, USA) 1:100. Phospho-S6 primary antibody (\#2211 polyclonal; Cell Signaling Technology, USA) 1:400 with FITC-labeled secondary antibody (Jackson ImmunoResearch Laboratories, USA) 1:100. Slides were mounted using SlowFade Gold antifade reagent with DAPI (S36939; Invitrogen, USA). Anti-GLEPP1 mouse monoclonal antibody (1B4) at 1:100 (Wiggins laboratory) with immunoperoxidase Vectastain Elite ABC kit (PK-6100; Vector Laboratories Inc, USA).

WT1 or PAX8/Ki67 double label analysis. In each section cells were characterized as to whether they were podocytes (WT1 positive nuclei) or PECs (PAX8 positive nuclei) according to specific markers and whether they were non-podocyte glomerular tuft cells, periglomerular or interstitial cells or tubular cells according to their position in relation to other cortical structures. Within these compartments cells whose nuclei were Ki67 positive were counted for $\mathrm{Fa} / \mathrm{fa}$ (6week), fa/fa (6week), $\mathrm{Fa} / \mathrm{fa}$ (30week), fa/fa (30week), Fa/fa (45week) and fa/fa (45week) groups $(n=5-6)$. Averaged values for the 12 consecutive images were used for each rat. Thus in the case of WT1 and PAX8 cell nuclei would be double-labelled for WT1/Ki67 or PAX8/Ki67 respectively, or in the case of other compartments would be labelled by Ki67 alone. Double immunofluorescent labeling was performed on $1.5 \mathrm{um}$ thick sections using the cell cycle marker Ki67 (Cat \# NB600-1252, Novus Biologicals, USA) and either the podocyte nuclear marker WT1 or the parietal cell (PEC) marker PAX8 (Cat \# ab53490, Abcom, USA) with DAPI (blue) staining. Compound images from the red, green, and blue channels for 12 consecutive glomerular profiles were made for each rat at 200x magnification with a glomerulus in the center of the field.

Glomerulosclerosis index. Estimated by a blinded observer using AZAN-stained histologic sections as $\%$ glomerulosclerosis for 30-50 consecutive glomeruli.

Urinary IGF1, IGF2 and serum insulin. $24 \mathrm{hr}$ urinary IGF1 and IGF2 excretion were measured using Rat IGF1 and IGF2 ELISA kit (Cat No. MBS824704 and MBS824828, MyBioSource.com, USA). Serum insulin was evaluated using Mercodia Rat Insulin ELISA kit (Mercodia AB, Sweden).

Statistical analysis. GraphPad PRISM software, version 6.0 (GraphPad Software, Inc., USA) was used. Supplemental Table 1 data are expressed as mean $\pm S D$, other data as mean \pm SEM. Differences among two groups were tested using the Mann-Whitney $U$ test, and more than two groups were tested using the Kruskal-Wallis test. When the result of the Kruskal-Wallis test was significant, a Dunn test was performed for post hoc analysis. Correlations between parameters were compared using Spearman's rank correlation coefficient analysis. Correlation P values were calculated using Stata 15 I/C (College Station, TX). A $P$-value $<0.05$ was considered statistically significant.

Received: 4 June 2019; Accepted: 16 October 2019;

Published online: 06 December 2019 


\section{References}

1. American Diabetes Association. Executive summary: Standards of medical care in diabetes-2014. Diabetes Care 37(Suppl 1), S5-13 (2014).

2. Stanton, R. C. Clinical challenges in diagnosis and management of diabetic kidney disease. Am J Kidney Dis 63(2 Suppl 2), S3-21 (2014).

3. Ogurtsova, K. et al. IDF Diabetes Atlas: Global estimates for the prevalence of diabetes for 2015 and 2040. Daibetes Res Clin Pract 28, $40-50$ (2017).

4. Masakane, I. et al. An overview of regular dialysis treatment in Japan (As of 31 December 2013). Ther Apher Dial 19, 540-574 (2015).

5. Alder, A. I. et al. Development and progression of nephropathy in type 2 diabetes: the United Kingdom Progressive Diabetes Study (UKPDS 64). Kidney Int 63, 225-232 (2003).

6. Tang, S. C., Leung, J. C. \& Lai, K. N. Diabetic tubulopathy: an emerging entity. Contrib Nephrol 170, 124-134 (2011).

7. Brosius, F. C. \& Coward, R. J. Podocytes, signaling pathways, and vascular factors in diabetic kidney disease. Adv Chronic Kidney Dis 21, 304-310 (2014)

8. Wiggins, R. C. The spectrum of podocytopathies: a unifying view of glomerular diseases. Kidney Int 71, 1205-1214 (2007).

9. Wharram, B. L. et al. Podocyte depletion causes glomerulosclerosis: diphtheria toxin-induced podocyte depletion in rats expressing human diphtheria toxin receptor transgene. J Am Soc Nephrol 16, 2941-2952 (2005).

10. Sato, Y. et al. Urine podocyte mRNAs mark progression of renal disease. J Am Soc Nephrol 20, 1041-52 (2009).

11. Fukuda, A. et al. Angiotensin II dependent persistent podocyte loss from destabilized glomeruli causes progression of end stage kidney disease. Kidney Int 81, 40-55 (2012).

12. Fukuda, A. et al. Growth-dependent podocyte failure causes glomerulosclerosis. J Am Soc Nephrol 23, 1351-1363 (2012).

13. Hodgin, J. B. et al. Glomerular aging and focal global glomerulosclerosis: A podometric perspective. J Am Soc Nephrol 26, 3162-3178 (2015).

14. Wickman, L. et al. Podocyte depletion in thin GBM and Alport Syndrome. PLoS One 11, e0155255 (2016).

15. Nishizono, R. et al. FSGS as an adaptive response to growth-induced podocyte stress. J Am Soc Nephrol 28, 2931-2945 (2017).

16. Fukuda, A. et al. Urinary podocyte and TGF- $\beta 1$ mRNA as markers for disease activity and progression in anti-glomerular basement membrane nephritis. Nephrol Dial Transplant 32, 1818-1830 (2017).

17. Pagtalunan, M. E. et al. Podocyte loss and progressive glomerular injury in type II diabetes. J Clin Invest 99, 342-348 (1997).

18. Meyer, T. W., Bennett, P. H. \& Nelson, R. G. Podocyte number predicts long-term urinary albumin excretion in Pima Indians with type II diabetes and microalbuminuria. Daibetologia 42, 1341-1344 (1999).

19. White, K. E. et al. Podocyte number in normotensive type I diabetic patients with albuminuria. Diabetes 51, 3083-3089 (2002).

20. Inoki, K. et al. mTORC1 activation in podocytes is a critical step in the development of diabetic nephropathy in mice. J Clin Invest 121, 2181-2196 (2011).

21. Gödel, M. et al. Role of mTOR in podocyte function and diabetic nephropathy in human and mice. J Clin Invest 121, 2197-2209 (2011).

22. Gassler, N. et al. Podocyte injury underlies the progression of focal segmental glomerulosclerosis in the fa/fa Zucker rat. Kidney Int 60, 106-16 (2001).

23. Kitada, M., Ogura, Y. \& Koya, D. Rodent models of diabetic nephropathy: their utility and limitations. Int J Nephrol Renovasc Dis 9 , 279-290 (2016).

24. Betz, B. \& Conway, B. R. An update on the use of animal models in diabetic nephropathy research. Curr Diab Rep 16, 18 (2016)

25. Fukuda, A. et al. Urine podocin:nephrin mRNA ratio (PNR) as a podocyte stress biomarker. Nephrol Dial Transplant 27, 4079-4087 (2012).

26. Ohlsson, C. et al. The role of liver-derived insulin-like growth factor-1. Endocrine reviews 30, 494-535 (2009).

27. Finkel, T. \& Pan, H. Key proteins and pathways that regulate lifespan. J Biol Chem 292, 6452-6460 (2017).

28. Sun, X., Chen, W.-D. \& Wang, Y.-D. DAF-16/FOXO transcription factor in aging and longevity. Frontiers Pharmacol 3, 548 (2017).

29. Kamenicky, P. et al. Growth hormone, insulin-like growth factor-1, and the kidney: pathophysiological and clinical implications. Endocr Rev 35, 234-281 (2014).

30. Kim, S. G., Buel, G. R. \& Blenis, J. Nutrient regulation of the mTOR complex 1 signaling pathway. Mol Cells 35, 463-473 (2013).

31. Goberdhan, D. C., Wilson, C. \& Harris, A. L. Amino acid sensing by mTORC1: Intracellular transporters mark the spot. Cell Metab 23, 580-589 (2016).

32. Inoki, K. \& Huber, T. Mammalian target of rapamycin signaling in the podocyte. Curr Opin Nephrol Hypertens 21, 251-257 (2012).

33. Kriz, W. \& Lemley, K. V. A potential role for mechanical forces in the detachment of podocytes and the progression of CKD. J Am Soc Nephrol 26, 258-69 (2015).

34. Ding, F. et al. Accelerated podocyte detachment and progressive podocyte loss from glomeruli with age in Alport syndrome. Kidney Int 92, 1515-1525 (2017).

35. Fukuda, A. et al. Urine podocyte mRNAs mark disease activity in IgA nephropathy. Nephrol Dial Transplant 30, 1140-1150 (2015).

36. Naik, A. S. et al. Quantitative podocyte parameters predict human native kidney and allograft half-lives. JCI Insight 1, e86943 (2016).

37. Szeto, C. C. et al. Messenger RNA expression of glomerular podocyte markers in the urinary sediment of acquired proteinuric disease. Clin Chim Acta 361, 182-190 (2005).

38. Wang, G. et al. Messenger RNA expression of podocyte-associated molecules in the urinary sediment of patients with diabetic nephropathy. Nephron Clin Pract 106, c169-c179 (2007).

39. Hara, M. et al. Apical cell membranes are shed into urine from injured podocytes: a novel phenomenon of podocyte injury. $J$ Am Soc Nephrol 16, 408-416 (2005).

40. Hara, M., Yanagihara, T. \& Kihara, I. Cumulative excretion of urinary podocytes reflects disease progression in IgA nephropathy and Scho"nlein-Henoch purpura nephritis. Clin J Am Soc Nephrol 2, 231-238 (2007).

41. Hara, M. et al. Urinary podocalyxin is an early marker for podocyte injury in patients with diabetes: establishment of a highly sensitive ELISA to detect urinary podocalyxin. Diabetologia 55, 2913-2919 (2012).

42. Hara, M. et al. Mitotic catastrophe causes podocyte loss in the urine of human diabetes. Am J Pathol 189, 248-257 (2019).

43. D’Agati, V. D. et al. Obesity-related glomerulopathy: clinical and pathologic characteristics and pathogenesis. Nat Rev Nephrol 12, 453-71 (2016).

44. Chang, A. R. et al. Bariatric surgery is associated with improvement in kidney outcomes. Kidney Int 90, 164-71 (2016).

45. Nehus, E. \& Mitsnefes, M. Childhood obesity and the metabolic syndrome. Pediatr Clin N Am 66, 31-43 (2019).

46. Gonzalez Suarez, M. et al. Diabetic nephropathy: Is it time yet for routine kidney biopsy? World J Diabetes 4, 245-255 (2013).

47. Sharma, S. et al. The modern spectrum of renal biopsy findings in patients with diabetes. Clin Am J Soc Nephrol 8, 1718-1724 (2013).

48. Steffes, M. W. et al. International Diabetic Nephropathy Study Group: Glomerular cell number in normal subjects and in type 1 diabetic patients. Kidney Int 59, 2104-2113 (2001).

49. Dalla Vestra, M. et al. Is podocyte injury relevant in diabetic nephropathy? Studies in patients with type 2 diabetes. Diabetes $\mathbf{5 2}$, 1031-1035 (2003).

50. Bilous, R. W. et al. Mean glomerular volume and rate of development of diabetic nephropathy. Diabetes 38, 1142-1147 (1989).

51. Wang, Y. et al. COL4A3 gene variants and diabetic disease in MODY. Clin J Am Soc Nephrol 13, 1162-1171 (2018).

52. Venkatareddy, M. et al. Estimating podocyte number and density in a single histologic section. J Am Soc Nephrol 25, 1118-1129 (2014). 
53. Allard, J. \& Duan, C. IGF-binding proteins: Why do they exist and why are there so many? Front Endocrinol 9, 1-11 (2018).

54. Saxton, R. A. \& Sabatini, D. M. mTOR signaling in growth, metabolism, and disease. Cell 168, 960-976 (2017).

55. Fine, L. The biology of renal hypertrophy. Kidney Int 29, 619-634 (1986).

56. Doi, T. et al. Progressive glomerulosclerosis develops in transgenic mice chronically expressing Growth hormone and Growth hormone releasing factor but not in those expressing Insulinlike growth factor-1. Am J Pathol 131, 398-403 (1988).

57. Feld, S. \& Hirschberg, R. Insulinlike growth factor1 and the kidney. Trends Endocrinol Metab 7, 85-93 (1996).

\section{Acknowledgements}

We thank Su Q. Wang and Mahboob Chowdhury for technical assistance. We acknowledge the research helping by Ms. Ofuku, Kawano, Otsu, Kawagoe, Nagata and Mr. Yamasaki, and the secretarial assistance by Ms. Tahara. A.F. acknowledges the support of research grant from the JSPS KAKENHI (Grant Number JP16K19198), the Japan Foundation for Applied Enzymology (Grant Number 15V005), the Mitsubishi Tanabe Pharma Corporation (Grant Number MTPS20160507013) and Takeda Science Foundation. R.W. was supported by the National Institutes of Health (grants R01 DK46073 and 102643).

\section{Author contributions}

A.F., R.W. and S.F. designed the experiments. A.M. and A.F. performed the experiments. A.M., A.F., Y.S., M.K. and K.K. analyzed the data. A.F. and R.W. wrote the manuscript. All authors approved the final version of the manuscript.

\section{Competing interests}

The authors declare no competing interests.

\section{Additional information}

Supplementary information is available for this paper at https://doi.org/10.1038/s41598-019-54692-z.

Correspondence and requests for materials should be addressed to A.F.

Reprints and permissions information is available at www.nature.com/reprints.

Publisher's note Springer Nature remains neutral with regard to jurisdictional claims in published maps and institutional affiliations.

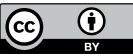

Open Access This article is licensed under a Creative Commons Attribution 4.0 International License, which permits use, sharing, adaptation, distribution and reproduction in any medium or format, as long as you give appropriate credit to the original author(s) and the source, provide a link to the Creative Commons license, and indicate if changes were made. The images or other third party material in this article are included in the article's Creative Commons license, unless indicated otherwise in a credit line to the material. If material is not included in the article's Creative Commons license and your intended use is not permitted by statutory regulation or exceeds the permitted use, you will need to obtain permission directly from the copyright holder. To view a copy of this license, visit http://creativecommons.org/licenses/by/4.0/.

(C) The Author(s) 2019 